\title{
Photoacoustic Trace Detection of Methane Using Compact Solid-State Lasers ${ }^{\dagger}$
}

\author{
Geng-Chiau Liang, Hon-Huei Liu, and A. H. Kung \\ Institute of Atomic and Molecular Sciences, Academia Sinica, P. O. Box 23-166, Taipei 106, Taiwan
}

\author{
A. Mohacsi,, A. Miklos, and P. Hess* \\ Institute of Physical Chemistry, University of Heidelberg, Im Neuenheimer Feld 253, \\ D-69120 Heidelberg, Germany
}

Received: April 3, 2000; In Final Form: June 1, 2000

\begin{abstract}
Methane was excited in the near-infrared (NIR) region using a pulsed grazing-incidence periodically poled lithium niobate optical parametric oscillator (OPO) and modulated diode laser radiation at $1650 \mathrm{~nm}$ as light sources and detected photoacoustically employing single tube and double tube acoustic resonators coupled with acoustic filters. The output power of the OPO was $\sim 60 \mathrm{~mW}$ at the acoustic resonance frequency of 2.12 $\mathrm{kHz}$, and therefore much higher than that of the diode laser radiation with $2 \mathrm{~mW}$. By combining the more powerful OPO with the more sensitive differential cell with two resonator tubes (sensitivity: $\alpha_{\min } \mathrm{W}=1.2 \times$ $10^{-9} \mathrm{~cm}^{-1} \mathrm{~W}$ ), an ultimate sensitivity of $20-30$ parts per billion (ppbV) is estimated for methane detection with the signal beam of the OPO in the near-infrared and sub-ppbV sensitivity in the mid-infrared region with the idler beam.
\end{abstract}

\section{Introduction}

Development of compact and yet sensitive devices for monitoring and analyzing trace gases has been of primary importance in applications such as pollution monitoring, biomedical sensing, plant physiology and plant growth analysis, and atmospheric research. Photoacoustic spectroscopy (PAS), based on the photoacoustic effect, in which the absorption of modulated light by a gaseous compound results in the generation of sound waves, has been identified as an ultrasensitive technique. ${ }^{1-3}$ Tunable solid-state light sources, such as diode lasers (DL) and optical parametric oscillators (OPO) combined with photoacoustic (PA) detectors, seem to be ideal tools for high-sensitivity trace-gas measurements. The compactness of solid-state devices offers the possibility of convenient mobile field measurements. ${ }^{4,5}$

The detection of methane based on the accidental coincidence between an absorption line and the $3.392 \mu \mathrm{m}$ HeNe laser transition was proposed as early as $1965 .{ }^{6}$ Excitation of methane with an OPO as a tunable light source first was used in vibrational energy transfer studies, clarifying the conditions for efficient relaxation of the excitation energy into translational energy, generating the PA effect. ${ }^{7,8}$ Recent interest in trace gas analysis led to the application of these sources in OPO-PA detectors allowing the investigation of several methane absorptions in the $3.3 \mu \mathrm{m}$ band. ${ }^{9}$ Four absorption lines were resolved in the corresponding PA spectrum of an $18 \mathrm{ppmV}$ (parts per million by volume) methane-synthetic air mixture. The sensitivity of the measurement was estimated as $1 \mathrm{ppmV}$.

Recently, sensitive photoacoustic detection of trace gases was achieved with a singly resonant continuous-wave (CW) OPO based on periodically poled lithium niobate (PPLN). ${ }^{10}$ The coarse tuning of the wavelength was obtained in this case by

\footnotetext{
† Part of the special issue "C. Bradley Moore Festschrift".

$\doteqdot$ JATE University, Department of Quantum Electronics, P. O. Box 406 , H-6701 Szeged, Hungary.
}

selection of a particular quasi-phasematching grating using a translator to move the crystal. Finer tuning was possible by changing the crystal temperature and/or the pump laser frequency. The CW OPO laser beam was modulated with a stabilized chopper. The sample gas, ethane, was excited in the fundamental IR region with an idler power of $40 \mathrm{~mW}$ at 3.34 $\mu \mathrm{m}$. A sensitivity of $0.5 \mathrm{ppbV}$ was reported in ref 10 , extrapolated from a single measurement. CW OPOs offer low background and low power fluctuations. However, the complex tuning and stabilization mechanism required limits the potential utility of this device in mobile field applications and the analysis of mixtures.

Diode-laser photoacoustic (DL-PA) measurements of methane absorption in the near-infrared (NIR) spectral region at 1660 $\mathrm{nm}$ were reported in ref 11 . The advantage of NIR diodes is that they work at room temperature, the disadvantage of the NIR spectral range is the low absorption coefficient of overtone and combination bands. The modulated laser power of the diode used was only about $0.7 \mathrm{~mW}$ in these first experiments, resulting in a minimum detectable concentration of methane of $60 \mathrm{ppmV}$. Later on an external cavity diode laser (ECDL) system was employed for measuring the PA spectrum of methane absorptions in the $1330 \mathrm{~nm}$ range. ${ }^{12}$ Despite the even smaller absorption coefficient in this spectral region, an improved sensitivity of $10 \mathrm{ppmV}$ was obtained with $3.1 \mathrm{~mW}$ modulated diode laser power. For many industrial applications this may be sufficient, but for methane detection in the atmosphere (methane is an anthropogenic greenhouse gas with a concentration of $1.7 \mathrm{ppmV}$ ) a sensitivity of at least $100 \mathrm{ppbV}$ is needed.

One of the requirements of sensitive detection is the implementation of a compact photoacoustic cell that works in the flow mode, since polar gases such as ammonia adsorb strongly at the walls. In addition, the cell design reduces external electromagnetic disturbances. ${ }^{13}$ Another one is the improvement of a compact pulsed grazing incidence optical parametric oscillator (GIOPO). This grazing-incidence cavity design has 


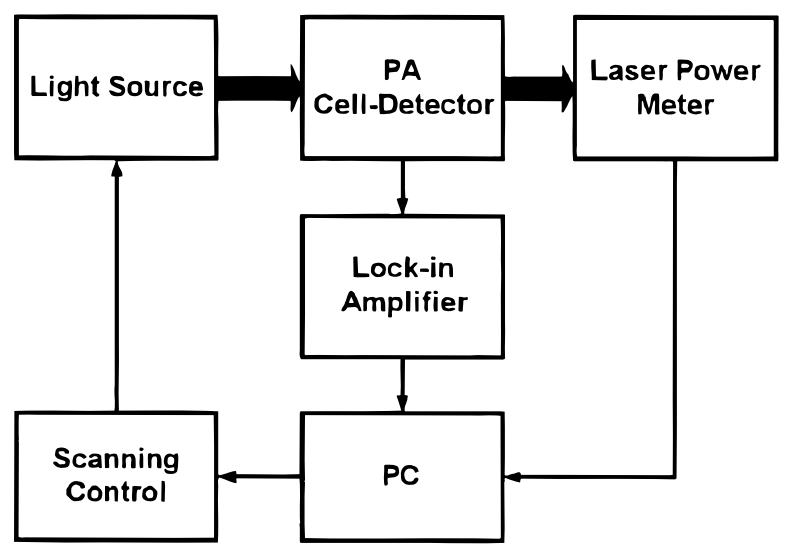

Figure 1. Schematic diagram of the photoacoustic detection setup.

sufficient power, high efficiency, broad and simple continuous tuning, and a line width that matches well with pressurebroadened gas line widths at one atmosphere. ${ }^{14}$ Its kilohertz repetition rate matches well with modulated $\mathrm{CW}$ laser PA detection. The compactness of the combined GIOPO and differential PA detector makes such a device very attractive for field applications in multi-gas emission monitoring and medical sensing. In this paper we report on the photoacoustic detection of methane using the NIR signal beam of a GIOPO and compare these results with those obtained with a diode laser working also near $1650 \mathrm{~nm}$. The results show that a sensitivity in the 20-30 ppbV level can be achieved with the OPO and an optimally designed PA detector even in the NIR spectral region.

\section{Experimental Section}

A block diagram of the typical setup used in the PA experiments is shown in Figure 1. Two sets of experiments were performed. In the first setup (OPO-PA), the light source was a grazing-incidence periodically poled lithium niobate OPO that is similar to that reported in ref 14 . This GIOPO was operated with the signal wavelength tuned to around $1650 \mathrm{~nm}$ in the NIR spectral region. The output power was $\sim 60 \mathrm{~mW}$ at $2.12 \mathrm{kHz}$, corresponding to a pulse energy of $28 \mu \mathrm{J}$. The line width of the signal was $\sim 0.2 \mathrm{~cm}^{-1}$. In addition, there was not more than 7 $\mathrm{mW}$ of broadband amplified parametric emission from the OPO ${ }^{14}$ Coarse tuning of the OPO was achieved by setting the temperature of the PPLN crystal and choosing the crystal grating period. Continuous fine scanning was accomplished by rotating a single mirror in the OPO cavity with a micro-stepping motor attached to a micrometer, controlled by a PC. The full width at half-maximum (fwhm) power scanning range for a single temperature and crystal grating period was $\sim 30 \mathrm{~cm}^{-1}$.

In this set of OPO experiments a simple PA cell with a lower sensitivity was used, which was a predecessor of the advanced differential cell described in detail below. It had a similar design except that it had only a single resonator tube with its first longitudinal acoustic resonance at $2.12 \mathrm{kHz}$. The cell was filled with a premixed methane/nitrogen mixture, and all measurements were performed under static conditions. Since the methane pressure in the closed cell was constant and the calibration plot was linear, no adsorption on the walls could be detected with the present sensitivity.

In the second setup (DL-PA), the light source was a $10 \mathrm{~mW}$ CW Thomson FP diode laser radiating near $1650 \mathrm{~nm}$. This laser emitted three modes, from which only the weakest coincided with a methane absorption line. The modulated power of this diode laser emission wavelength was about $1 \mathrm{~mW}$. A certified mixture of $100 \mathrm{ppmV}$ methane in nitrogen was diluted by

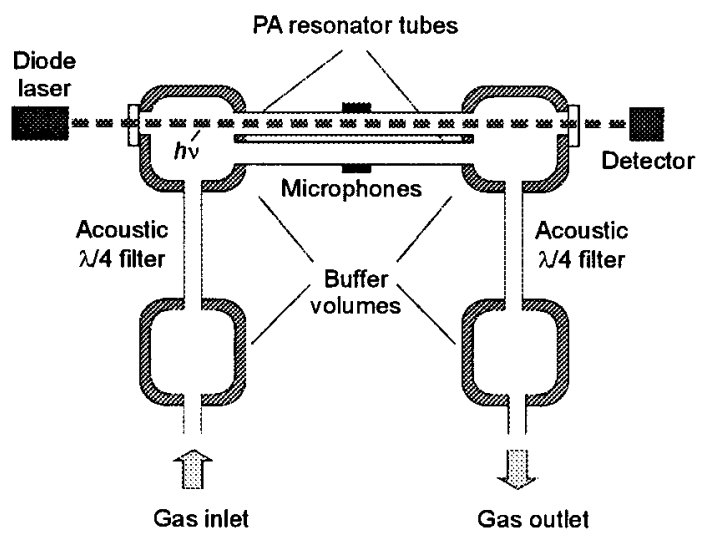

Figure 2. Diagram showing the design of a fully symmetric photoacoustic cell suitable for flow measurements.

additional nitrogen using mass flow controllers (Tylan, FC-260). In both sets of experiments, the PA signal was measured by a dual phase lock-in amplifier (Stanford SR series) and evaluated by a PC. The background signal was measured using pure nitrogen, while the flow and electromagnetic noise were determined by blocking the laser beam.

The differential PA detector employed here was specifically designed for fast time response, low acoustic and electric noise characteristics, and high sensitivity. To reduce the flow noise and external electromagnetic disturbances from electronic devices a fully symmetrical design with two resonators was developed. Two resonant acoustic cells $(5.5 \mathrm{~mm}$ diameter resonator tubes) were placed between two acoustic filters (Figure 2 ). The gas flow passes through both tubes, producing about the same flow noise in both resonator tubes. However, the laser light irradiates only one of them, thus the PA signal is generated in only one tube. A microphone (Knowles 3029) was placed in the central section of each tube flush with the wall and the PA signal was amplified by a differential amplifier. The two microphones have the same sensitivity around the acoustic resonance frequency of the tubes at $4 \mathrm{kHz}$. Thus all noise components, which are coherent in the two tubes, are effectively suppressed. In the present experiments flow rates between 0.3 $\mathrm{L} / \mathrm{min}$ and $0.8 \mathrm{~L} / \mathrm{min}$ were chosen.

\section{Results}

1. OPO-PA Measurements. The GIOPO was scanned for several concentrations of methane in the region of $1650 \mathrm{~nm}$. A representative measured spectrum is shown in Figure 3. The spectral resolution was determined by the OPO line width. This spectrum covering $80 \mathrm{~cm}^{-1}$ was obtained by concatenating wavelength scans of the GIOPO at three adjacent temperatures of the PPLN crystal. From the spectrum it is found that the PA signal is strongest at the $\mathrm{Q}$ branch of the $2 v_{3}$ overtone vibrational mode of methane at the wavelength indicated by an arrow in Figure 3. PA methane concentration measurements were then made at this wavelength. The background signal measured for pure nitrogen was about $1.5 \mu \mathrm{V}$ and the noise signal was 250 $\mathrm{nV}$ (time constant: $1 \mathrm{~s}$ ). The methane concentration was reduced in several steps from 12600 to $300 \mathrm{ppbV}$. The PA signal, after subtracting off the constant background signal, is plotted against the methane concentration in Figure 4. This curve, which calibrates the trace detection of methane for this system, shows a linear fitting correlation of 1.0045 over more than four decades of methane concentration. The noise signal of $250 \mathrm{nV}$ determines the limiting detectivity of the present setup to $\sim 0.1 \mathrm{ppmV}$ at unity signal-to-noise ratio $(\mathrm{SNR} \approx 1)$. 


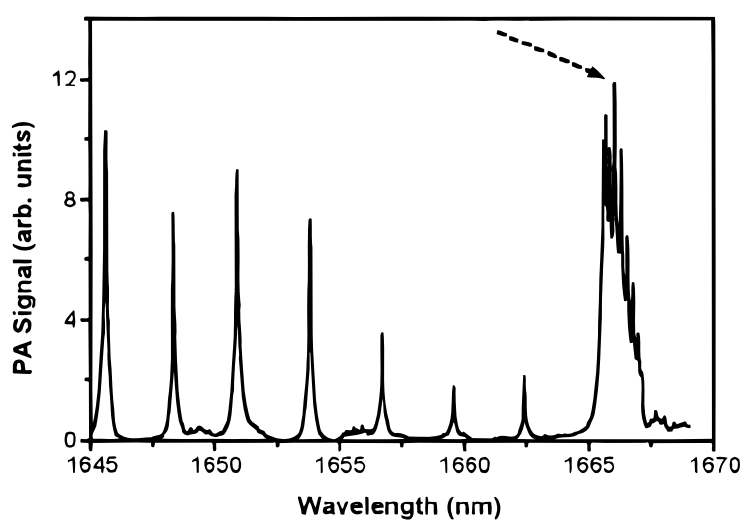

Figure 3. Power-normalized photoacoustic spectrum obtained by scanning the GIOPO near the Q branch of the $2 v_{3}$ vibrational mode of methane. The methane concentration was $880 \mathrm{ppmV}$. The arrow indicates the absorption peak at which concentration measurements were made.

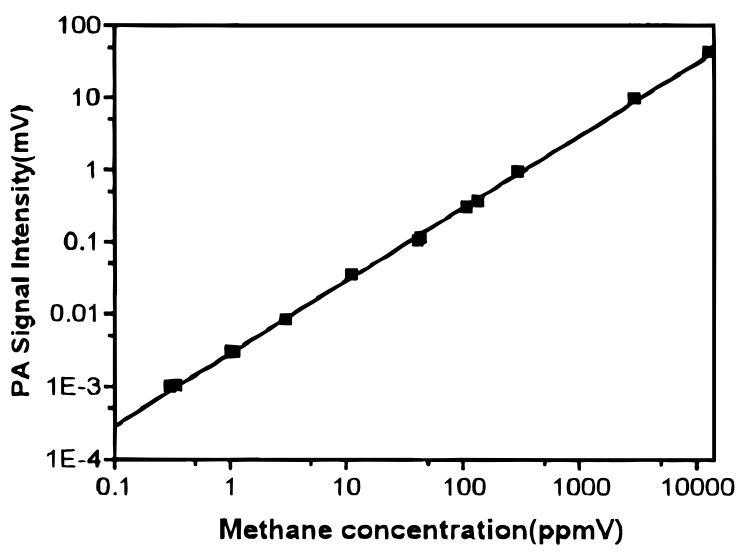

Figure 4. Photoacoustic signal versus methane/nitrogen concentration in the OPO-PA experiment. Squares are measured data. The solid line is a linearly fitted curve to the data. A background signal of $1.25 \mu \mathrm{V}$ has been subtracted from the raw PA signal. Note that the vertical scale and the horizontal scale are both logarithmic.

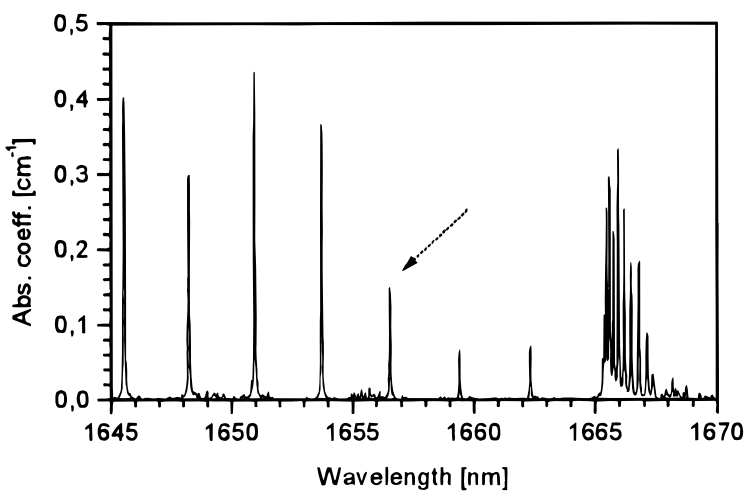

Figure 5. Calculated absorption spectrum of methane near the $\mathrm{Q}$ branch of the $2 v_{3}$ vibrational mode. The diode laser emission overlaps with one of the weaker lines as indicated.

2. DL-PA Results. In these experiments the absorption of a selected mode of the $10 \mathrm{~mW}$ Thomson FP diode laser at 1650 nm generated the PA signal. The exact absorption peak was located by a temperature scan of the laser diode. Only one absorption line at $1656.55 \mathrm{~nm}\left(6036.65 \mathrm{~cm}^{-1}\right)$ of the methane overtone band could be covered within the tunability range of the diode with a modulated power of $1 \mathrm{~mW}$. Figure 5 shows the methane absorption spectrum of this spectral region calculated from the HITRAN database. The arrow in this figure indicates the coinciding line. The value obtained for the

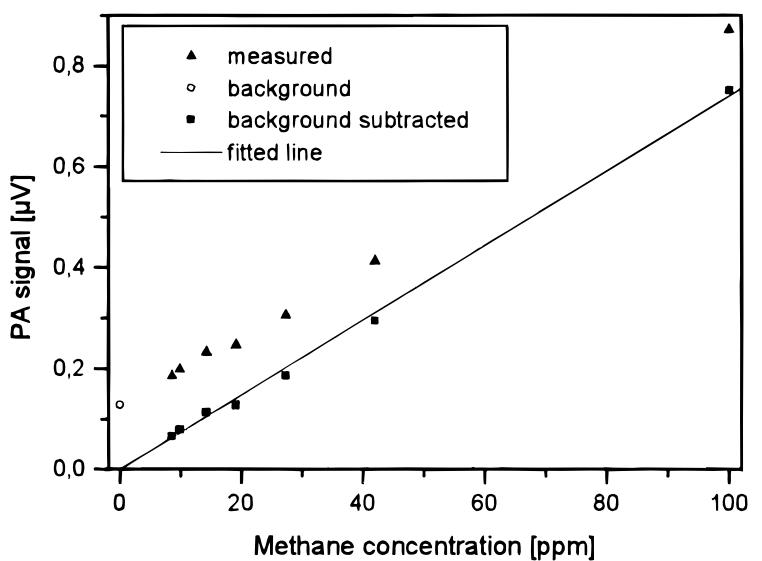

Figure 6. Photoacoustic signal versus methane/nitrogen concentration in the DL-PA experiment.

absorption coefficient at the peak of this line is $0.147 \mathrm{~cm}^{-1}$. The calculated HITRAN spectrum agrees well with the measured PA spectrum given in Figure 3.

First the noise performance of the cell was checked without gas flow. The intrinsic noise of the miniature electret microphone was very large; a value of $15.7 \mu \mathrm{V}$ was measured for the broadband noise. However the correlated noise measured by the lock-in with the laser beam blocked was only $\sim 40 \mathrm{nV}$, which corresponds to a methane concentration of $3 \mathrm{ppmV}$. The noise increased slightly $(\sim 20 \%)$ at $1 \mathrm{~L} / \mathrm{min}$ flow rate. The background signal measured with the laser on for pure nitrogen was about three times this $(120 \mathrm{nV})$. The measured concentration of $8 \mathrm{ppmV}$ corresponds to a signal/background ratio of one $(\mathrm{SBR}=1)$.

A calibration curve was determined by measuring the PA signal for different methane concentrations. The methane content was reduced in several steps from 100 to $8 \mathrm{ppmV}$. Since the background was relatively stable in these experiments, it could be subtracted from the measured PA signal. In this way a nearly linear calibration curve was found in the 8-100 ppmV concentration range as exhibited in Figure 6. The correlation coefficient of the linear fit was better than 0.995 .

\section{Discussion}

First we discuss the sensitivity achieved in modulated diode laser photoacoustics and compare the results with absorption measurements using this light source. The limiting sensitivity of the more sensitive differential PA detector can be characterized by the following expression: $\alpha_{\min } \mathrm{W}=1.2 \times 10^{-9} \mathrm{~cm}^{-1} \mathrm{~W}$, where $\alpha_{\min }$ and $\mathrm{W}$ are the smallest measured absorption coefficient (in $\mathrm{cm}^{-1}$ ) and the modulated incident laser power (in Watts), respectively. ${ }^{10}$ This value can be compared with the sensitivity of PA detectors used by other authors. For example, the sensitivities of the PA detectors used in intracavity photoacoustic measurements with a $\mathrm{CO}_{2}$ laser ${ }^{15}$ and an ion laser pumped dye laser ${ }^{16}$ were $1.8 \times 10^{-8}$ and $2 \times 10^{-8} \mathrm{~cm}^{-1} \mathrm{~W}$, respectively. The best extracavity single path detectors also had sensitivities between $1 \times 10^{-8}$ and $2 \times 10^{-8} \mathrm{~cm}^{-1} \mathrm{~W} .{ }^{1}$ Thus, the optimized differential detector has about 1 order of magnitude higher sensitivity. It is of interest to note that extracavity multipass schemes reach a comparable sensitivity of $\alpha_{\text {min }} \mathrm{W} \approx 10^{-9} \mathrm{~cm}^{-1} \mathrm{~W}$, whereas with the present cell it would be possible to further improve the sensitivity with a multipass arrangement.

In the following, the sensitivity of photoacoustics, using a small cell, will be compared with the results of single-path 
optical absorption measurements of methane. Since absorption experiments are usually performed with an extended optical path length, we take the concentration-path-length product to compare the sensitivities. Diode-laser absorption measurements in the $1660 \mathrm{~nm}$ band have been reported in ref 11,17 . The detection limits reported at $\mathrm{SNR}=1$ were $1.15 \mathrm{ppmVm}$ (parts per million volume meter $)^{11}$ and $300 \mathrm{ppbVm} .{ }^{15}$ Taking into account that the length of the acoustic resonator in the DL-PA measurements was only $4 \mathrm{~cm}$, the measured $8 \mathrm{ppmV}$ methane concentration $(\mathrm{SBR}=1)$ corresponds to $\sim 0.32 \mathrm{ppmVm}$. Since the laser power used in ref 17 was $\sim 7$ and $2 \mathrm{~mW}$ (unmodulated for a conservative estimate) in the present measurements, another factor of 3.5 would be gained in PA measurements yielding a sensitivity of about $90 \mathrm{ppbVm}(\mathrm{SBR}=1)$ for the same power and path length as in ref 17. It should be pointed out, however, that to some extent such a comparison of the sensitivity of the two methods is academic, since photoacoustics is better suited for small compact cells and absorption experiments are reasonable if long optical path lengths are available.

PA detection with NIR diode lasers will be further improved, as diodes with higher output become available, because the signal is proportional to laser power below the level where saturation occurs. This, however, is not the case for absorption measurements. For industrial applications, where a sensitivity in the parts per million range is sufficient, the most suitable method can be selected. A sensitivity in the parts per billion range, on the other hand, would require photoacoustic detection and a NIR diode laser power above $100 \mathrm{~mW}$.

Pulsed PA detection is of great interest because a quantitative theoretical treatment of the PA signal is possible for high Q resonators making calibration unnecessary. ${ }^{18,19}$ The sensitivity to be expected for the sensitive differential PA detector combined with a pulsed laser source can be estimated by introducing an equivalent laser pulse energy instead of the modulated laser power. The equivalent pulse energy can be determined by the following expression: ${ }^{18,19} E=W_{\mathrm{L}} Q / \omega$, where $E$ is the pulse energy in joules, $W_{\mathrm{L}}$ is the modulated power in Watts, $Q$ is the quality factor of the acoustic resonator (in this case $Q=18)$ and $\omega$ is the angular modulation frequency $(\omega=$ $2 \pi x 4000=25000 \mathrm{~s}^{-1}$ ). This means that $1 \mathrm{~mW}$ modulated power corresponds to $0.72 \mu \mathrm{J}$ pulse energy. From the detection limit of the DL-PA measurements of $8 \mathrm{ppmV}$ at $1 \mathrm{~mW}$ modulated power, based on the background of $120 \mathrm{nV}$, it can be extrapolated that the differential PA detector would be able to detect $\sim 20$ to $30 \mathrm{ppbV}$ methane with $28 \mu \mathrm{J}$ pulse energy.

The OPO results reported here were taken at the Q branch of the $2 v_{3}$ overtone band which is quite congested. In real situations, such as the investigation of mixtures, detection of a single rovibrational transition will be a better choice. As can be seen in Figure 3, the improvement in selectivity can be substantial at a $<20 \%$ sacrifice to the overall sensitivity.

Note that the detection limit usually has to be defined by the signal-to-background (S/B) rather than the signal-to-noise $(\mathrm{S} / \mathrm{N})$ ratio (which is common practice), because the background is always present when a light pulse hits the PA cell and may not be constant. Since the background in pulsed photoacoustics normally is larger than in the modulated cw mode, the practical sensitivity is expected to be somewhat lower in pulsed systems. An optimally designed OPO, on the other hand, could have higher power ${ }^{14}$ so that a further improvement of the sensitivity is within reach. In the pulsed mode the signal increase, however, may be somewhat smaller than the expected linear behavior if optical saturation comes into play at higher pulse energies. A pulsed OPO also has higher broadband background emission which may limit the ultimate selectivity and sensitivity that can be reached with such a system if time-gating is not efficient.

A pulsed continuously tunable BBO OPO previously has been applied for recording photoacoustic absorption spectra of acetylene..$^{20}$ The narrow-band radiation $(\sim 0.3 \mathrm{GHz})$ was used to excite rovibrational lines of a combination band in the NIR spectral region with the $3 \mathrm{~ns} 865.5 \mathrm{~nm}$ idler pulses containing $2 \mathrm{~mJ}$ pulse energy. However, no concentration measurement was performed in this spectroscopic work.

The idler of the pulsed GIOPO, on the other hand, radiates in the IR with a pulse energy of $40 \mu \mathrm{J}$ and a bandwidth of 0.9 $\mathrm{cm}^{-1}$. Measurements are planned with this idler beam to take advantage of the considerably higher transition dipole moments in the mid-infrared. These experiments should reach a sensitivity comparable to those with the CW PPLN OPO, especially when the idler bandwidth can be reduced to the $0.1 \mathrm{~cm}^{-1}$ level by resonating the idler instead of the signal wavelength in the GIOPO. This change will not influence the operation, i.e., the tuning ability of the GIOPO. Yet, the pulsed GIOPO offers fast and relatively simple tuning which is crucial for detecting gas mixtures in portable remote systems.

\section{Conclusions}

A very sensitive and compact differential PA cell with two resonator tubes was tested using a room temperature operated NIR diode laser with milliwatt modulated power. The sensitivity of about $8 \mathrm{ppmV}$ realized with this setup demonstrates the suitability of photoacoustics for industrial trace gas detection, especially if a short optical path length is required. The sensitivity will increase proportional to the laser power as more powerful NIR laser diodes become available. A first pulsed photoacoustic study on methane detection was performed with a grazing-incidence periodically poled lithium niobate OPO employing a less sensitive photoacoustic detector with only one resonator tube. The sensitivity estimated for the combination of the more sensitive differential cell with the signal beam of the pulsed GIOPO in the NIR spectral range is $20-30 \mathrm{ppbV}$. With the idler beam in the mid-infrared it should be roughly a factor of 90 higher according to the strong increase in the absorption coefficient.

Acknowledgment. Partial financial support of this work by the German-Hungarian bilateral scientific cooperation project (No.UNG-016-97), the China Petroleum Corporation, and the Fonds der Chemischen Industrie is gratefully acknowledged.

\section{References and Notes}

(1) Sigrist, M. W. Air Monitoring by Spectroscopic Techniques, Wiley: New York, 1994; pp 163-238.

(2) Harren, F.; Reuss, J. Encyclopedia of Applied Physics, VCH: Weinheim, 1997; pp 413-435.

(3) Miklos, A.; Hess, P. Anal. Chem. 2000, 72, 30A.

(4) Tittel, F. K., Ed.; Special Issue, Appl. Phys. B 1998, 67, No.3\&4.

(5) Bohren, A.; Sigrist, M. W. Infrared Phys. Technol. 1997, 38, 423.

(6) Moore, C. B. Appl. Opt. 1965, 4, 252.

(7) Hess, P.; Moore, C. B. J. Chem. Phys. 1976, 65, 2339. 5525 .

(8) Hess, P.; Kung, A. H.; Moore, C. B. J. Chem. Phys. 1980, 72,

(9) Debuisschert, T.; Bozoki, Z.; Miklos, A.; Raffy, J.; Huber, A.; Pocholle, J. P. Proc. SPIE-Int. Soc. Opt. Eng. 1995, 2506 (Air Pollution and Visibility Measurements), 720.

(10) Kühnemann, F.; Schneider, K.; Martis, A. A. E.; Urban, W.; Schiller, S.; Mlynek, J. Appl. Phys. B 1998, 66, 741.

(11) Schaefer, S.; Mashni, M.; Sneider, J.; Miklos, A.; Hess, P.; Pitz, H.; Pleban, K.-U.; Ebert, V. Appl. Phys. B 1998, 66, 511. 
(12) Miklos, A.; Hess, P.; Romolini, A.; Spada, C.; Lancia, A.; Kamm, S.; Schafer, S. Photoacoustic and photothermal phenomena: 10th International Conference; Scudieri, F., Bertolotti, M., Eds. American Institute of Physics: Woodbury, USA, 1999; 217-219.

(13) Miklos, A.; Hess, P.; Mohacsi, A.; Sneider, J.; Kamm, S.; Schafer, S. Photoacoustic and photothermal phenomena: 10th International Conference; Scudieri, F., Bertolotti, M., Eds. American Institute of Physics: Woodbury, USA, 1999; 126-128.

(14) Yu, Chi-Sheng; Kung, A. H. J. Opt. Soc. Am. B 1999, 16, 2233.
(15) Harren, F. J. M.; Bijnen, F. G. C.; Reuss, J.; Voesenek, L. A. C. J.; Blom, C. W. P. M. Appl. Phys. B. 1990, 50, 137. (16) Davidson, J.; Gutow, J. H.; Zare, R. N. J. Phys. Chem. 1990, 94, 4069-4073.

(17) Uehara, K.; Tai, H. Appl. Opt. 1992, 31, 809.

(18) Schaefer, S.; Miklos, A.; Hess, P. Appl. Opt. 1997, 36, 3202.

(19) Schaefer, S.; Miklos, A.; Pusel, A.; Hess, P. Chem. Phys. Lett. 1998, $285,235$.

(20) Johnson, M. J.; Haub, J. G.; Orr, B. J. Opt. Lett. 1995, 20, 1277. 